\title{
EXPERIMENTOS BIOLÓGICOS CON SERES HUMANOS EN EL MARCO DEL DERECHO DE LOS CONFLICTOS ARMADOS
}

\section{BIOLOGICAL EXPERIMENTS WITH HUMAN BEINGS IN THE FRAMEWORK OF ARMED CONFLICT LAW}

\author{
Dra. Ana Cristina GALLEGO HERNÁNDEZ ${ }^{1}$
}

\begin{abstract}
Resumen: En situaciones de conflicto armado, los Convenios de Ginebra prohíben la práctica de experimentos biológicos o científicos, excepto cuando estén justificados médicamente. En este sentido, los principios éticos y jurídicos de asistencia a la salud continúan rigiendo las actuaciones del personal de salud, quienes ostentan siempre el deber de proteger la salud física y mental de las personas, así como el de aliviar su sufrimiento, de conformidad con el derecho.
\end{abstract}

Abstract: In situations of armed conflict, the Geneva Conventions prohibit the practice of biological or scientific experiments, except when they are medically justified. In this sense, the ethical and legal principles of health care continue to govern the actions of health personnel, who always have the duty to protect the physical and mental health of people and alleviate their suffering in accordance with the right.

Palabras clave: experimentos biológicos, conflictos armados, grupos vulnerables, Convenio de Ginebra, ética, consentimiento.

Keywords: Biological experiments, armed conflicts, vulnerable groups, Geneva Convention, ethics, consent.

\footnotetext{
${ }^{1}$ Profesora Doctora de Derecho Internacional Público, Centro Universitario San Isidoro, adscrito a la Universidad Pablo de Olavide. El presente estudio se enmarca en el Proyecto I+D Excelencia 2015, referencia DER2015-65906-P y en el Proyecto I+D+i Europa Investigación EUIN2017-85437. aagallegoo@ gmail.com.
} 
IUS ET SCIENTIA (ISSN: 2444-8478) 2019, Vol.5, nº 1, pp. 90-111

EXPERIMENTOS BIOLÓGICOS CON SERES HUMANOS EN EL MARCO DEL DERECHO DE LOS

CONFLICTOS ARMADOS, Ana Cristina GALLEGO HERNÁNDEZ Centro Universitario San Isidoro

(Universidad Pablo de Olavide). aagallegoo@gmail.com

Recibido: 14/01/2019. Aceptado: 16/05/2019 DOI: http://dx.doi.org/10.12795/IETSCIENTIA.2019.i01.04

\section{Los experimentos biológicos: concepto y principios}

Sin reparar en tecnicismos, los experimentos biológicos consisten en las investigaciones de carácter médico que, de conformidad con la ética profesional, se realiza en seres humanos, con el objetivo de que los resultados obtenidos generen un beneficio social. Como es lógico y de común conocimiento, el médico ${ }^{2}$ es el sujeto especializado y capacitado para prevenir, diagnosticar y tratar las enfermedades humanas y, por lo tanto, su función radica en velar por la buena salud y el mejor interés de sus pacientes. Con el propósito de progresar en las intervenciones preventivas, en los diagnósticos y en los tratamientos terapéuticos, así como para comprender las causas y efectos de las enfermedades; la investigación científica en los propios seres humanos, en muchas ocasiones, resulta una fase fundamental para generar nuevos conocimientos.

En cualquier caso, el fin general de la promoción de la buena salud, evidentemente, entra en colisión con los riesgos y daños que pueden recaer en el sujeto que colabora en la investigación. Es por ello, que los experimentos deben supeditarse a unas normas y estándares jurídicos y éticos, para que los riesgos sean mínimos o inexistentes y para que se proteja la vida, la salud, la dignidad y la intimidad, tanto individual como de la generalidad. Por lo que en ningún caso se puede justificar que se realicen o permitan actos de tortura u otras formas de tratos crueles, inhumanos o degradantes ${ }^{3}$. En otras palabras y en el sentido jurídico estricto, las prácticas señaladas deberán realizarse garantizándose el derecho a la vida (y, en consonancia, con el derecho a la salud) y la prohibición de que se realicen torturas o tratos degradantes.

\footnotetext{
2 "La función del personal de salud es proteger la salud física y mental y aliviar los sufrimientos, proporcionando los cuidados con humanidad, respetando la dignidad del paciente y sin discriminación". BADIA MARTÍ, A., "Asistencia humanitaria. Reflexiones desde la perspectiva jurídica internacional de los principios humanitarios y éticos”, Revista Electrónica IUS ET SCIENTIA, n², vol. 2, 2016, pp. 10.

${ }^{3}$ RODRÍGUEZ-VILLASANTE Y PRIETO, J. L., "Principios éticos de la asistencia sanitaria en tiempos de conflicto armado y situaciones de emergencia", Revista de Investigación y Educación en Ciencias de la Salud, n 2, vol. 2, 2017, p. 82.
} 
IUS ET SCIENTIA (ISSN: 2444-8478) 2019, Vol.5, nº 1, pp. 90-111

EXPERIMENTOS BIOLÓGICOS CON SERES HUMANOS EN EL MARCO DEL DERECHO DE LOS

CONFLICTOS ARMADOS, Ana Cristina GALLEGO HERNÁNDEZ Centro Universitario San Isidoro

(Universidad Pablo de Olavide). aagallegoo@gmail.com

Recibido: 14/01/2019. Aceptado: 16/05/2019 DOI: http://dx.doi.org/10.12795/IETSCIENTIA.2019.i01.04

De conformidad con la evolutiva protección de los derechos humanos que se viene desarrollando, desde el fin de la Segunda Guerra Mundial y, en concreto, en lo que respecta a los experimentos médicos permitidos y a la garantía de los derechos señalados con anterioridad, "las relaciones del paciente (o participante ${ }^{4}$ ) con el personal sanitario están presididos por los principios de la bioética: consentimiento informado del paciente, imparcialidad y no discriminación, así como la confidencialidad respecto a la intimidad del paciente"

Asimismo, fruto del Código de Nuremberg ${ }^{6}$ y de la tan revisada Declaración de Helsinki ${ }^{7}$, este tipo de investigaciones deben cumplir con las siguientes pautas o principios ${ }^{8}$ básicos:

- Como se viene señalando, el médico o personal de atención de la salud debe velar por la vida y la buena salud del sujeto que trata. Por lo que los conocimientos deben

\footnotetext{
${ }^{4}$ Comúnmente denominado "cobaya humana".

5 De conformidad con "os códigos éticos sanitarios y cristalizados en la Declaración Universal sobre "Bioética y Derechos Humanos" de la UNESCO de 19 d octubre de 2005”. BADIA MARTÍ, A., "Asistencia humanitaria...”, op. cit., p. 10.

${ }^{6} 6$ Conjunto de principios elementales, recopilados tras el análisis de los casos juzgados por el Tribunal Internacional de Núremberg, que han servido de inspiración a importantes instrumentos posteriores. Código de Núremberg. Experimentos médicos permitidos. Disponible en:
} http://www.bioeticanet.info/documentos/Nuremberg.pdf Consultado el 10 de octubre de 2018.

${ }^{7}$ Declaración de Helsinki de la AMM- Principios éticos para las investigaciones médicas en seres humanos. Adoptada por la $18^{\mathrm{a}}$ Asamblea Médica Mundial, Helsinki, Finlandia, junio 1964 y enmendada por: la 29a Asamblea Médica Mundial, Tokio, Japón, octubre 1975, 35ª Asamblea Médica Mundial, Venecia, Italia, octubre 1983, 41ª Asamblea Médica Mundial, Hong Kong, Septiembre 1989, 48 a Asamblea General Somerset West, Sudáfrica, octubre 1996, 52 asamblea General, Edimburgo, Escocia, octubre 2000, Nota de Clarificación, agregada por la Asamblea General de la AMM, Washington 2002, Nota de Clarificación, agregada por la Asamblea General de la AMM, Tokio 2004, 59ª Asamblea General, Seúl, Corea, octubre 2008 y $64^{\mathrm{a}}$ Asamblea General, Fortaleza, Brasil, octubre 2013.

${ }^{8}$ Los principios señalados tienen un fuerte y esencial carácter ético, pero el necesario y evidente respeto justifica que se hayan recogido en múltiples documentos jurídicos y políticos, tanto internos como internacionales. Por ejemplo en España Real Decreto para regular los ensayos clínicos, los Comités de Ética de la Investigación y el Registro Español de Estudios Clínicos (Real Decreto 1090/2015, de 4 de diciembre, por el que se regulan los ensayos clínicos con medicamentos, los Comités de Ética de la Investigación con medicamentos y el Registro Español de Estudios Clínicos, BOE 307, 24 de diciembre de 2015), se encuentra inspirado en dichas pautas e instrumentos. 
IUS ET SCIENTIA (ISSN: 2444-8478) 2019, Vol.5, nº 1, pp. 90-111

EXPERIMENTOS BIOLÓGICOS CON SERES HUMANOS EN EL MARCO DEL DERECHO DE LOS

CONFLICTOS ARMADOS, Ana Cristina GALLEGO HERNÁNDEZ Centro Universitario San Isidoro

(Universidad Pablo de Olavide). aagallegoo@ gmail.com

Recibido: 14/01/2019. Aceptado: 16/05/2019 DOI: http://dx.doi.org/10.12795/IETSCIENTIA.2019.i01.04

subordinarse al cumplimiento de dicha obligación.

- Con motivo de que "el progreso de la medicina se basa en la investigación que, en último término, debe incluir estudios en seres humanos", ven subordinados al cumplimiento de principios éticos que permiten, como no puede ser de otro modo, la garantía del derecho a la vida, del derecho a la salud y de los derechos humanos, en general. De manera que, la búsqueda de nuevos conocimientos se somete a la más escrupulosa protección de dichos derechos.

- La investigación médica sobre seres humanos debe realizarse por profesionales "competentes y cualificados apropiadamente".

- La justificación de dicha investigación exige que la importancia del objetivo supere los riesgos y costos, lo cuales deben estar cuantificados, ser mínimos y ser posteriormente remediados, si llegara a generar perjuicio.

- Los objetivos ${ }^{10}$, el método y las fuentes deben quedar claramente detallados y justificados en un proyecto de investigación, el cual debe ser aprobado y controlado en toda fase de su procedimiento por un comité de ética de investigación.

- Aunque en la actualidad resulte evidente, es considerado un logro el requisito ineludible de que se exigiera a los sujetos intervinientes el previo consentimiento libre y voluntario $^{11}$ tras la recepción de la información suficiente y adecuada ${ }^{12}$. Dicha máxima es

\footnotetext{
${ }^{9}$ Declaración de Helsinki de la AMM- Principios éticos para las investigaciones médicas en seres humanos-, $64^{\mathrm{a}}$ Asamblea General, Fortaleza, Brasil, octubre 2013, p. 2.

10 "An experiment not serving a therapeutic purpose should al- ways be regarded as prohibited". MEBRING, S., "Medical war crimes”, Max Planck UNYB 15, 2011, p. 249.

${ }^{11}$ Artículo 7 del Pacto Internacional de Derechos Civiles y Políticos (PIDCP), adoptado por la Asamblea General de las Naciones Unidas mediante la Resolución 2200 A (XXI), de 16 de diciembre de 1966. Entró en vigor el 23 de marzo de 1976. España los ratificó el 27 de abril de 1977, BOE n 103, de 30 de abril de 1977.

12 "The consent of the person "treated" to the procedures can never be used as a defense". MEBRING, S., 
IUS ET SCIENTIA (ISSN: 2444-8478) 2019, Vol.5, nº 1, pp. 90-111

EXPERIMENTOS BIOLÓGICOS CON SERES HUMANOS EN EL MARCO DEL DERECHO DE LOS

CONFLICTOS ARMADOS, Ana Cristina GALLEGO HERNÁNDEZ Centro Universitario San Isidoro

(Universidad Pablo de Olavide). aagallegoo@gmail.com

Recibido: 14/01/2019. Aceptado: 16/05/2019 DOI: http://dx.doi.org/10.12795/IETSCIENTIA.2019.i01.04

recogida en el artículo 7 del Pacto Internacional de Derechos Civiles y Políticos.

- La investigación solo podrá hacerse sobre grupos vulnerables si se justifica que no pueden ser objeto del experimento sujetos que no requieren de una especial protección ${ }^{13}$.

- Y entre otros, durante tanto las distintas fases de experimento como con posterioridad, se debe garantizar la intimidad y confidencialidad de aquellos sujetos que se prestan a la consecución del mismo.

En cualquier caso, como base a la "ética de investigación", el personal dedicado y capacitado para realizar experimentos biológicos, en todo momento debe actuar de conformidad con los principios (jurídicos) de humanidad e imparcialidad ${ }^{14}$, de manera que no solo no permita el sufrimiento humano, sino que lo prevenga y alivie; y para que preste la asistencia sanitaria en todo momento y sin discriminación.

\section{¿Prohibición absoluta de los experimentos médicos, científicos o biológicos en situaciones de conflictos armados? La excepción que confirma la regla}

Como ocurrió a mediados del siglo pasado en los casos que han inspirado al desarrollo de los códigos éticos de la asistencia de la salud, en ocasiones, el personal dedicado a la misma ha aprovechado situaciones de inestabilidad para el desarrollo de experimentos sin

op.cit., p. 249.

${ }^{13}$ Si la talidomida se hubiera probado debidamente en embarazadas, el uso generalizado del mismo para remediar las náuseas no hubiera provocado miles de malformaciones y, por tanto, perjuicios.

${ }^{14}$ BADIA MARTÍ, A., "Comentarios a los "Principios éticos de la asistencia de salud en tiempo de conflictos armados y otras situaciones de emergencia", Comité Internacional Cruz Roja, 2015”, Revista de Bioética y Derecho \& Perspectivas Bioéticas, nº 36, 2016, p. 131. 
IUS ET SCIENTIA (ISSN: 2444-8478) 2019, Vol.5, nº 1, pp. 90-111

EXPERIMENTOS BIOLÓGICOS CON SERES HUMANOS EN EL MARCO DEL DERECHO DE LOS

CONFLICTOS ARMADOS, Ana Cristina GALLEGO HERNÁNDEZ Centro Universitario San Isidoro

(Universidad Pablo de Olavide). aagallegoo@gmail.com

Recibido: 14/01/2019. Aceptado: 16/05/2019 DOI: http://dx.doi.org/10.12795/IETSCIENTIA.2019.i01.04

cumplir las garantías o en detrimento de las partes contrarias ${ }^{15}$. Es por ello que, el Derecho Internacional Humanitario prohíbe expresamente, aunque con pequeñas matizaciones, los experimentos médicos o científicos en situaciones de conflicto armado ${ }^{16}$, tanto internacionales como de índole interno. Es decir, cuando exista un enfrentamiento armado y regido por los Convenios de Ginebra, al igual que en otras situaciones de emergencia, los principios éticos y jurídicos de asistencia a la salud, seguirán rigiendo las actuaciones del personal de salud, quienes como se ha señalado, ostentan siempre el deber de proteger la salud física y mental de las personas, así como el de aliviar su sufrimiento, de conformidad con el derecho. Es por estos motivos, que dichos sujetos (principalmente médicos, pero también enfermeros, farmacéuticos y otros) no podrán hacer uso de los privilegios, que además específicamente los Convenios de Ginebra les reconoce, para fines distintos de la estricta protección de la salud.

Los Convenios de Ginebra reconocen de manera expresa la prohibición generalizada de que, en situaciones de conflictos armados, nacionales o internacionales, no cabe el desarrollo de "experimentos biológicos" o "experimentos médicos o científicos" ". Ya

\footnotetext{
${ }^{15}$ In the Doctors' Trial of 1947 ( the case of the United States of America against Karl Brandt and 22 other accused) "the criminal experiments were high altitude, freezing, malaria, mus- tard gas, sulfanilamide, bone, muscle and nerve regeneration, bone transplant, sea-water, epidemic jaundice, sterilization, typhus (and other vaccines), poison, and explosives experiments. Furthermore, R. Brandt and Sievers were specifically charged with the illegal endeavor of killing 112 Jewish persons for completing a skeleton collection for the Reich's University of Strasbourg". MEBRING, S., op. cit., p. 259.
}

${ }^{16}$ De acuerdo al Derecho Internacional Humanitario, no existe una definición sencilla y concreta del concepto de conflicto armado, no obstante, el Department of Peace and Conflict Research de la Universidad de Uppsal se posiciona al describirlo como: "An armed conflict is a contested incompatibility that concerns government and/or territory where the use of armed force between two parties, of which at least one is the government of a state, results in at least 25 battle-related deaths". Department of Peace and Conflict Research, Uppsala University. Disponible en: http://www.pcr.uu.se/research/ucdp/definitions/ Consultado el 10 de octubre de 2018.

${ }^{17}$ Sin reparar en el uso variable de "biológico" o "médico o científico": en los artículo 12 del I Convenio de Ginebra para aliviar la suerte que corren los heridos y los enfermos de las fuerzas armadas en campaña, y del II Convenio de Ginebra para aliviar la suerte que corren los heridos, los enfermos y los náufragos de las fuerzas armadas en el mar; artículo 13 del III Convenio de Ginebra relativo al trato debido a los prisioneros de guerra; artículo 32 del IV Convenio de Ginebra relativo a la protección debida a las personas civiles en tiempo de guerra. Todos ellos aprobados el 12 de agosto de 1949 por la Conferencia Diplomática para Elaborar Convenios Internacionales destinados a proteger a las víctimas de la guerra, celebrada en Ginebra 
IUS ET SCIENTIA (ISSN: 2444-8478) 2019, Vol.5, nº 1, pp. 90-111

EXPERIMENTOS BIOLÓGICOS CON SERES HUMANOS EN EL MARCO DEL DERECHO DE LOS

CONFLICTOS ARMADOS, Ana Cristina GALLEGO HERNÁNDEZ Centro Universitario San Isidoro

(Universidad Pablo de Olavide). aagallegoo@gmail.com

Recibido: 14/01/2019. Aceptado: 16/05/2019 DOI: http://dx.doi.org/10.12795/IETSCIENTIA.2019.i01.04

que, como ocurrió en épocas pasadas, dichas prácticas pueden ser utilizadas para generar trato degradantes, tortura o incluso para progresar en la propia investigación sin escrúpulos éticos.

Así las cosas, se considera contrario al Derecho general y una violación del Derecho Internacional Humanitario, y por lo tanto, una infracción grave; cualquier experimento médico o científico que "no esté indicado por su estado de salud y [que no se lleve a cabo] de acuerdo con las normas médicas generalmente reconocidas, las cuales se aplicarían en análogas circunstancias médicas, a los nacionales de la Parte que realiza el acto" ${ }^{\text {18 }}$. En otras palabras, es una violación grave del Derecho las investigaciones científicas en seres humanos que el personal de salud lleve a cabo, voluntaria, imprudentemente y con conocimiento de que ello supone un grave peligro para la salud de la persona o personas objeto del experimento ${ }^{19}$. No obstante, para que constituya dicha violación grave, es preciso que la víctima siempre se encuentre en poder de una parte diferente ${ }^{20}$.

del 12 de abril al 12 de agosto de 1949. Entrada en vigor: 21 de octubre de 1950. Respectivamente, 75 UNTS 31, 75 UNTS 85, 75 UNTS 135 \& 75 UNTS 287. Convenio para mejorar la suerte de los heridos y enfermos de las Fuerzas Armadas en campaña, Ginebra, España se adhesiona el 12 agosto 1949, BOE de 23 agosto 1952. Convenio para mejorar la suerte de los heridos, enfermos y náufragos de las Fuerzas Armadas en el mar. Ginebra, España se adhesiona el 12 agosto 1949, BOE de 26 agosto 1952. Convenio relativo al trato de los prisioneros de guerra. Ginebra, España se adhesiona el 12 agosto 1949, BOE de 5 septiembre 1952. Convenio relativo a la protección de personas civiles en tiempo de guerra. Ginebra, España se adhesiona el 12 agosto 1949, BOE de 2 septiembre 1952.

18 Artículo 11 del Protocolo I adicional a los Convenios de Ginebra de 1949 relativo a la protección de las víctimas de los conflictos armados internacionales, 8 de junio de 1977. En vigor de forma general desde el 7 de diciembre de 1978. 1125 UNTS 3. En vigor para España desde el 21 de octubre de 1989, BOE n $^{\circ} 241$, de 7 de octubre de 1989.

19 “Unlawfulness, willingness and knowledge”. MEBRING, S., op. cit., p. 236.

20 "This restriction was included to ensure the sovereignty of parties to a conflict over their own nationals. [...] The Rome Statute, for example, determines that a perpetrator does not have to know the nationality of his victim; solely that he belongs to the adverse party. Hence, a physician's act can incur prosecution when he treats a patient who is not of the same party to the conflict as he himself and he knows this. This broad interpretation of civilian protected persons is within the object and purpose of the drafters of the Geneva Conventions. Nonetheless, care should be taken not to broaden the concept beyond practical applicability.", ibid. 
IUS ET SCIENTIA (ISSN: 2444-8478) 2019, Vol.5, nº 1, pp. 90-111

EXPERIMENTOS BIOLÓGICOS CON SERES HUMANOS EN EL MARCO DEL DERECHO DE LOS

CONFLICTOS ARMADOS, Ana Cristina GALLEGO HERNÁNDEZ Centro Universitario San Isidoro

(Universidad Pablo de Olavide). aagallegoo@gmail.com

Recibido: 14/01/2019. Aceptado: 16/05/2019 DOI: http://dx.doi.org/10.12795/IETSCIENTIA.2019.i01.04

Por lo que, de conformidad con lo descrito y en aras de proteger a los distintos sujetos "vulnerables" en un conflicto armado, la prohibición expresa de realizar experimentos biológicos a seres humanos se ve violada cuando se lleva a cabo un acto contrario a la salud; cuando dicho acto tiene una relación directa o indirecta con un conflicto (ya sea internacional o nacional); y cuando el autor o autores del hecho son conscientes de que su actuación es una violación grave contrario al derecho o, al menos, que no cumple con los principios éticos y profesionales sobre los que se rige su profesión. Esto es, suponen una grave violación los experimentos biológicos que causan intencionalmente graves sufrimientos o lesiones ${ }^{21}$ (que no son consecuencia del propio conflicto armado) en una persona objeto de protección ${ }^{22}$.

\section{Regulación y consecuencia jurídica de la práctica de experimentos biológicos en situaciones de conflicto armado}

Tal y como se ha ido referenciando a largo del trabajo, los distintos instrumentos que conforman el Derecho Internacional Humanitario hacen suficientes alusiones a la práctica de experimentos biológicos o médicos a seres humanos para comprender que, a priori, es una actividad prohibida por el Derecho de los conflictos armados.

De los cuatro Convenios de Ginebra y de los dos Protocolos ${ }^{23}$, la explicación más

21 "the Elements of Crimes, biological experiments seriously endanger the physical or mental health or integrity of the persons subjected to them when they are non-therapeutic, not justified by medical reason". MEBRING, S., op. cit., p. 246.

22 "The "classic" grave breaches are "wilful killing, torture or inhuman treatment, including biological experiments, wilfully causing great suffering or serious injury to body or health" 45 of the protected persons by an individual in the respective Convention. All these crimes can also be committed by a physician in a medical con- text. For a medical act or procedure by a physician to be prosecuted as a grave breach of the Conventions, the act must also meet the requirements of the grave breaches under the Conventions". Ibid.

${ }^{23}$ El Protocolo II adicional a los Convenios de Ginebra de 1949 relativo a la protección de las víctimas de los conflictos armados sin carácter internacional, de 12 de agosto de 1977, no realiza referencia expresa a los 
IUS ET SCIENTIA (ISSN: 2444-8478) 2019, Vol.5, nº 1, pp. 90-111

EXPERIMENTOS BIOLÓGICOS CON SERES HUMANOS EN EL MARCO DEL DERECHO DE LOS

CONFLICTOS ARMADOS, Ana Cristina GALLEGO HERNÁNDEZ Centro Universitario San Isidoro

(Universidad Pablo de Olavide). aagallegoo@gmail.com

Recibido: 14/01/2019. Aceptado: 16/05/2019 DOI: http://dx.doi.org/10.12795/IETSCIENTIA.2019.i01.04

detallada y extensiva se realiza en el ya referido artículo 11 del Protocolo $\mathrm{I}^{24}$, el cual determina como principio general que "se prohíben en particular, aunque medie el consentimiento de las referidas personas: (...) b) los experimentos médicos o científicos". Dicha prohibición, es igualmente reproducida en los tres primeros Convenios ${ }^{25}$. No obstante, tanto IV Convenio para la protección de los Civiles, como el previamente citado Protocolo I, abren la puerta a una limitada excepción que confirma la regla del principio general: "Está estrictamente prohibido todo atentado contra (la) vida y (...) en particular matarlos o exterminarlos, someterlos a tortura, efectuar en ellos experimentos biológicos" ${ }^{26}$ excepto cuando estos sean "requeridos por el tratamiento médico de una persona protegida" 27 . Esto es, de puntillas intentando remediar la rotundidad de la prohibición, el Convenio IV y, de manera justificada, el relevante artículo 11 del Protocolo I, contemplan que: a) cuando el estado de salud lo requiera y justifique, b) de conformidad con las normas y principios éticos y científicos, c) y, en aras del interés personal; cabe que se lleven a cabo investigaciones e intervenciones científicas y médicas ${ }^{28}$.

experimentos biológicos, no obstante, ello no se interpreta de manera restrictiva, sino que se entiende que tampoco cabe la realización de investigación médica o científica en situaciones de conflicto interno, de acuerdo con el artículo 5.2.e.

${ }^{24}$ Protocolo I adicional a los Convenios de Ginebra de 1949 relativo a la protección de las víctimas de los conflictos armados internacionales, 8 de junio de 1977. En vigor de forma general desde el 7 de diciembre de 1978. 1125 UNTS 3. En vigor para España desde el 21 de octubre de 1989, BOE n ${ }^{\circ} 41$, de 7 de octubre de 1989.

${ }^{25}$ El análisis explicado se realiza sin tenerse en cuenta los motivos y el distinto uso empleado en los instrumentos del calificativo "biológico" y "médico o científico". Aunque en la declaración como violación de los experimentos, en los cuatro Convenios se utiliza la expresión "experimentos biológicos", en el reconocimiento de su prohibición solo se emplea en los dos primeros; mientras que los dos últimos y en el Primer Protocolo se califican como "experimentos médicos o científicos".

${ }^{26}$ Utilizo la rotundidad del artículo 12 del I y II Convenio, así como el artículo 12 del III Convenio.

${ }^{27}$ Expresión extraída del artículo 32 del IV Convenio.

${ }^{28}$ En parecidos términos, Mebring considera que: "Biological experiments are explicitly and separately named as a form of inhuman treatment. This proves the emphasis placed on the prohibi- tion of experiments 
IUS ET SCIENTIA (ISSN: 2444-8478) 2019, Vol.5, nº 1, pp. 90-111

EXPERIMENTOS BIOLÓGICOS CON SERES HUMANOS EN EL MARCO DEL DERECHO DE LOS

CONFLICTOS ARMADOS, Ana Cristina GALLEGO HERNÁNDEZ Centro Universitario San Isidoro

(Universidad Pablo de Olavide). aagallegoo@gmail.com

Recibido: 14/01/2019. Aceptado: 16/05/2019 DOI: http://dx.doi.org/10.12795/IETSCIENTIA.2019.i01.04

En este sentido, los tratados de Ginebra prohíben la práctica de experimentos biológicos o científicos sobre los seres humanos y, en especial, sobre los grupos vulnerables que a continuación se señalan. De manera que, si se incumple injustificadamente dicha negativa, la violación será considerada como una infracción grave ${ }^{29}$ de los distintos instrumentos, lo cual tiene como consecuencia jurídica que, de conformidad al Estatuto de Roma ${ }^{30}$-artículo $8^{\circ}$-, la Corte Penal Internacional puede calificar y considerar dicha infracción como crimen de guerra ${ }^{31}$, tanto en conflictos armados internos como internacionales.

En este sentido, los médicos o el personal de salud que participase en la comisión de dichas violaciones graves, deberán ser enjuiciados, caso por caso ${ }^{32}$, si es que no han preservado la salud y el interés del paciente, en todo momento. Por lo que, los elementos del crimen, como se ha venido desarrollando, consisten en que, en primer lugar, el acto, es decir, el experimento médico, haya causado un peligro físico o psíquico en la salud o en la

on protected persons in the system of the Conventions. Physicians are allowed to use new therapeutic methods if such treatment is medically justified, for the amelioration of the health of a patient, and the patient, if competent, has provided his informed con- sent.62 Whether something is a justified new therapeutic method or whether it is purely experimental may be controversial. A physician should thus always conduct a careful analysis as to whether a new pro- cedure is considered humane. The informed consent of a patient to the procedure is an important element of such an analysis although consent to an inhuman procedure can never justify said procedure". MEBRING, S., op.cit., p. 243.

${ }^{29}$ Artículo 50 del I Convenio; artículo 51 del II Convenio; artículo 130 del III Convenio; artículo 147 del IV Convenio. En relación a los Protocolos, solo se tiene en cuenta el primero de ellos que es el que se refiere a los experimentos biológicos y, al igual que con la prohibición, en el apartado $4^{\circ}$ del artículo 11 determina con más detalle las consecuencias jurídicas de dicha violación: "Constituirá infracción grave del presente Protocolo toda acción u omisión deliberada que ponga gravemente en peligro la salud o la integridad física o mental de toda persona en poder de una Parte distinta".

${ }^{30}$ Estatuto de Roma de la Corte Penal Internacional, A/CONF.183/9, de 17 de julio de 1998, enmendado por los procès verbaux de 10 de noviembre de 1998, 12 de julio de 1999, 30 de noviembre de 1999, 8 de mayo de 2000, 17 de enero de 2001 y 16 de enero de 2002. Dicho tratado entró en vigor el 1 de julio de 2002. España lo ratificó el 24 de octubre de 2000, (BOE 126, 27 de mayo de 2002).

${ }^{31}$ En opinión de Mebring, dichos actos constituyen crímenes de guerra médicos. "Medical war crimes can be defined as willful acts seri- ously endangering the health or integrity of a detained person who is affiliated with the adversary committed by physicians in carrying out their medical duties during an armed conflict". MEBRING, S., op.cit., p. 235.

${ }^{32}$ Ibid, p. 243. 
IUS ET SCIENTIA (ISSN: 2444-8478) 2019, Vol.5, nº 1, pp. 90-111

EXPERIMENTOS BIOLÓGICOS CON SERES HUMANOS EN EL MARCO DEL DERECHO DE LOS

CONFLICTOS ARMADOS, Ana Cristina GALLEGO HERNÁNDEZ Centro Universitario San Isidoro

(Universidad Pablo de Olavide). aagallegoo@gmail.com

Recibido: 14/01/2019. Aceptado: 16/05/2019 DOI: http://dx.doi.org/10.12795/IETSCIENTIA.2019.i01.04

integridad de civiles, enfermos, prisioneros, etc. Adicionalmente, dicha investigación se haya realizado sin razón o justificación médica y en contra del interés del sujeto ${ }^{33}$. Y, por último, que el sujeto o sujetos sobre los que se ocasiona el daño estén siendo tratados o estén en poder de una parte distinta o encontrada con las de su procedencia o condición. Todo ello sin que se tenga en cuenta la existencia o no de consentimiento por parte del sujeto sobre el que se realiza la acción, ya que como la Corte determinó en la elaboración del Estatuto, se considera que cualquier persona detenida no puede proporcionar un consentimiento válido para la ejecución del procedimiento ${ }^{34}$. En todo caso se entiende que habrá signos de coacción o intimidación.

Asimismo, como se recoge en el artículo 30 del Estatuto de Roma, los elementos señalados deben haberse desarrollado con conocimiento e intención. Esto quiere decir, que se excluyen de ser enjuiciados como crímenes de guerra los daños ocasionados involuntaria, imprudente o negligentemente. Del mismo modo, el sujeto infractor, ya sea civil o militar, tiene que conocer que existe un conflicto armado y la condición de la víctima $^{35}$ o sujetos sobre el que se realiza el experimento (recuérdese que estos suelen pertenecer a grupos vulnerables). ¿Cuándo podrán enjuiciarse dichos experimentos como violaciones graves a los convenios de Ginebra? Como recoge el artículo 29 del Estatuto de Roma, el conocimiento por la Corte de los mismos podrá llevarse a cabo en cualquier

\footnotetext{
33 "The consent of the person "treated" to the procedures can never be used as a defense". MEBRING, S., op.cit., p. 249.

34 "Consent is not a defence to this crime. The crime prohibits any medical procedure which is not indicated by the state of health of the person concerned and which is not consistent with generally accepted medical standards which would be applied under similar medical circumstances to persons who are nationals of the party conducting the procedure and who are in no way deprived of liberty. This footnote also applies to the same element for article 8 (2) (b) (x)-2". Nota 46, Elements of Crimes. Disponible en: https://www.icccpi.int/NR/rdonlyres/336923D8-A6AD-40EC-AD7B-45BF9DE73D56/0/ElementsOfCrimesEng.pdf
}

Consultado el 10 de octubre de 2018.

${ }^{35}$ Ibid, p. 250. 
IUS ET SCIENTIA (ISSN: 2444-8478) 2019, Vol.5, nº 1, pp. 90-111

EXPERIMENTOS BIOLÓGICOS CON SERES HUMANOS EN EL MARCO DEL DERECHO DE LOS

CONFLICTOS ARMADOS, Ana Cristina GALLEGO HERNÁNDEZ Centro Universitario San Isidoro

(Universidad Pablo de Olavide). aagallegoo@ gmail.com

Recibido: 14/01/2019. Aceptado: 16/05/2019 DOI: http://dx.doi.org/10.12795/IETSCIENTIA.2019.i01.04

momento, ya que dichos crímenes son imprescriptibles ${ }^{36}$.

De manera complementaria a los Convenios de Ginebra y a sus Protocolos, en situación de conflicto armado, al Pacto internacional de Derechos Civiles y Políticos (artículo 7), para los tiempos de paz; y en un sentido similar al Estatuto de la Corte Penal Internacional, los instrumentos que confeccionan la puesta en marcha de los distintos tribunales internacionales ad hoc contemplan, directa e indirectamente, la prohibición de practicar experimentos biológicos o actos contrarios a la salud. En este sentido, el Estatuto del Tribunal Internacional para la ex Yugoslavia ${ }^{37}$ realiza una alusión directa a los Convenios de Ginebra y, en concreto, a que dicho Tribunal podrá enjuiciar a los responsables de “experimentos biológicos". Asimismo, tanto el Tribunal Penal Internacional de Ruanda ${ }^{38}$, como el Tribunal Especial para Sierra Leona ${ }^{39}$ reconocen directamente su competencia para procesar las violaciones de los Convenios de Ginebra (y, en concreto, el artículo 3), aunque en esta ocasión se hace referencia a la "mutilación" y no a los experimentos biológicos.

Complementariamente a lo señalado y desde diferentes perspectivas, también se han elaborado múltiples acuerdos e instrumentos de diversas disciplina y peso en los que se

\footnotetext{
${ }^{36}$ En el mismo sentido la imprescriptibilidad de los delitos era reconocido por el Estatuto del Tribunal Militar Internacional de Núremberg.

${ }^{37}$ Resolución 808 (1993) del Consejo de Seguridad de 22 de febrero de 1993, aprobada en su 3175ª sesión, S/ RES/ 808 (1993).

${ }^{38}$ Resolución 955 (1994) del Consejo de Seguridad de 8 de noviembre de 1994, aprobada en su 3453ª sesión, S/ RES/ 955 (1994).

39 "El 16 de enero de 2002 se firmó en Freetown (Sierra Leona) el Acuerdo entre las Naciones Unidas y el Gobierno de Sierra Leona acerca del Establecimiento de un Tribunal Especial para Sierra Leona, en cuyo anexo figuraba el Estatuto del Tribunal. El Acuerdo entró en vigor, de conformidad con su artículo 21, el 12 de abril de 2002, tras ser ratificado por Sierra Leona". United Nations Audiovisual Library of International Law. Disponible en: http://legal.un.org/avl/pdf/ha/scsl/scsl_s.pdf Consultado el 10 de octubre de 2018.
} 
IUS ET SCIENTIA (ISSN: 2444-8478) 2019, Vol.5, nº 1, pp. 90-111

EXPERIMENTOS BIOLÓGICOS CON SERES HUMANOS EN EL MARCO DEL DERECHO DE LOS

CONFLICTOS ARMADOS, Ana Cristina GALLEGO HERNÁNDEZ Centro Universitario San Isidoro

(Universidad Pablo de Olavide). aagallegoo@gmail.com

Recibido: 14/01/2019. Aceptado: 16/05/2019 DOI: http://dx.doi.org/10.12795/IETSCIENTIA.2019.i01.04

contempla la prohibición de dichas prácticas ${ }^{40}$.

\section{Los grupos vulnerables con necesidades especiales o con prioridades en cuestiones de salud}

Cuando la investigación o el experimento biológico, en situaciones de normalidad (es decir, en tiempo de paz) requiere o aconseja la práctica del mismo sobre personas pertenecientes a grupos vulnerables, tal y como se reconoce en las pautas éticas, tanto los investigadores como los comités de ética deberán asegurar la existencia de una protección específica que permita salvaguardar los derechos y el bienestar de dichos sujetos con características especiales.

"Según la Declaración de Helsinki, algunos grupos y personas vulnerables "pueden tener más posibilidades de sufrir abusos o daño adicional"41. Esto implica que la vulnerabilidad concierne elementos de juicio tanto sobre la probabilidad como sobre el grado de daño físico, psicológico o social, así como una mayor sensibilidad al engaño o la violación de la confidencialidad. Es importante reconocer que la vulnerabilidad concierne no solo la capacidad de dar el consentimiento inicial para participar en una investigación, sino también aspectos de la participación continua en estudios de investigación (...) Aunque los comités de ética de la investigación pueden requerir protecciones especiales solo para posibles participantes de forma colectiva para un proyecto en particular, los investigadores y otros involucrados en la investigación deben tener en cuenta aquellos factores que hacen vulnerable a cada participante individual y tomar medidas apropiadas para mitigar esos

\footnotetext{
40 Véase IHL database, customary IHL. Disponible en: https://ihl-databases.icrc.org/customaryihl/eng/docs/v2_rul_rule92 Consultado el 10 de octubre de 2018.

${ }^{41}$ Declaración de Helsinki de la AMM- Principios éticos para las investigaciones médicas en seres humanos-, 64a Asamblea General, Fortaleza, Brasil, octubre 2013, p. 4.
} 
IUS ET SCIENTIA (ISSN: 2444-8478) 2019, Vol.5, nº 1, pp. 90-111

EXPERIMENTOS BIOLÓGICOS CON SERES HUMANOS EN EL MARCO DEL DERECHO DE LOS

CONFLICTOS ARMADOS, Ana Cristina GALLEGO HERNÁNDEZ Centro Universitario San Isidoro

(Universidad Pablo de Olavide). aagallegoo@gmail.com

Recibido: 14/01/2019. Aceptado: 16/05/2019 DOI: http://dx.doi.org/10.12795/IETSCIENTIA.2019.i01.04

factores ${ }^{42}$.

En cualquier caso, en función de distintas características y variables (situación de conflicto armado o tiempo de paz; en función de las peculiaridades sociales ${ }^{43}$; en función de la capacidad $^{44}$; etc.), multitud de sujetos podrían llegar a calificarse como grupos vulnerables.

De manera general, los participantes que de conformidad a la capacidad para prestar su consentimiento (discapacitados psíquicos, menores...), que en función de las relaciones jerárquicas (militares, policía...), por la dependencia con el Estado (presos, ancianos en asilos, personas beneficiarias de asistencia social...) o con motivo de su situación particular de salud (enfermos, embarazadas, personas con debilidad física), entre otras razones, se consideren potencial o definitivamente vulnerables, no podrá asumir "más que riesgos mínimos" 45 y en un experimento científico. Y, por tanto, su participación deberá estar fundamentada, deberá existir una vigilancia particular por los comités de ética pertinentes y, si resultase necesario, se deberá complementar su consentimiento ${ }^{46}$ con el

\footnotetext{
${ }^{42}$ Consejo de Organizaciones Internacionales de las Ciencias Médicas y Organización Mundial de la Salud, "Pautas éticas internacionales para la investigación relacionada con la salud con seres humanos", Cuarta Edición. Ginebra: Consejo de Organizaciones Internacionales de las Ciencias Médicas (CIOMS); 2016, p. 65.
}

43 "Las personas también pueden ser vulnerables porque alguna de las circunstancias (temporales o permanentes) en que viven hace menos probable que otros estén atentos y se preocupen por sus intereses. Esto puede suceder con personas marginadas, estigmatizadas o que enfrentan exclusión social o prejuicio, lo que aumenta la probabilidad de que otros pongan sus intereses en riesgo, de manera intencional o no". Ibid.

44 "En algunos casos, las personas son vulnerables porque no tienen la capacidad relativa (o absoluta) de proteger sus propios intereses. Esto puede ocurrir cuando las personas tienen deficiencias relativas o absolutas en su capacidad decisoria, educación, recursos, fuerza u otros atributos necesarios para proteger sus propios intereses". Ibid.

${ }^{45}$ Consejo de Organizaciones Internacionales de las Ciencias Médicas y Organización Mundial de la Salud, "Pautas éticas internacionales para la investigación relacionada con la salud con seres humanos", Cuarta Edición. Ginebra: Consejo de Organizaciones Internacionales de las Ciencias Médicas (CIOMS); 2016, p. 67.

46 Así ocurre, especialmente, cuando en la investigación participan adultos sin capacidad para dar el consentimiento informado, niños o adolescentes. Véase, Consejo de Organizaciones Internacionales de las Ciencias Médicas y Organización Mundial de la Salud, "Pautas éticas internacionales para la investigación relacionada con la salud con seres humanos", Cuarta Edición. Ginebra: Consejo de Organizaciones 
IUS ET SCIENTIA (ISSN: 2444-8478) 2019, Vol.5, nº 1, pp. 90-111

EXPERIMENTOS BIOLÓGICOS CON SERES HUMANOS EN EL MARCO DEL DERECHO DE LOS

CONFLICTOS ARMADOS, Ana Cristina GALLEGO HERNÁNDEZ Centro Universitario San Isidoro

(Universidad Pablo de Olavide). aagallegoo@gmail.com

Recibido: 14/01/2019. Aceptado: 16/05/2019 DOI: http://dx.doi.org/10.12795/IETSCIENTIA.2019.i01.04

sujeto jurídicamente pertinente (tutor legal, familiar, etc).

En lo que respecta a la concreta situación de conflictos armados, de conformidad a los criterios descritos y al derecho vigente, en mi opinión, los grupos vulnerables pueden clasificarse de conformidad a la protección que brindan los Convenios de Ginebra, sus Protocolos y los instrumentos complementarios. En este sentido, un primer y evidente grupo vulnerable lo integran los militares heridos y enfermos (así como los náufragos), los cuales deberán ser respetados y asistidos con humanidad por la parte que los tenga en su poder. Del mismo modo, el "Convenio de Ginebra relativo al trato debido a los prisioneros de guerra" exige y prioriza el trato humano en toda circunstancia de dichos prisioneros (como grupo vulnerable), lo cual descarta cualquier tratamiento médico que no esté escrupulosamente justificado ${ }^{47}$. U, en tercer lugar, otro gran grupo vulnerable, por no decir el "gran grupo", le corresponde al conjunto de ciudadanos o nacionales no militares que no participan activamente en el conflicto. Dentro de éste, al mismo tiempo, podemos encontrar otros (sub)grupos con necesidades especiales o con prioridades de salud, como por ejemplo, las embarazadas ${ }^{48}$, los niños ${ }^{49}$, los discapacitados o personas con trastornos psíquicos o físicos, principalmente.

\section{La necesaria investigación médica en situaciones excepcionales}

\footnotetext{
Internacionales de las Ciencias Médicas (CIOMS); 2016, p. 69-75.

${ }^{47}$ Artículo 13 del III Convenio de Ginebra.

${ }^{48}$ Las mujeres embarazadas disfrutarán, en igual medida que los súbditos del Estado interesado, de todo trato preferente (artículo 38 del IV Convenio), ello sin perjuicio de que "las mujeres deben ser tratadas con todas las consideraciones debidas a su sexo" (artículos 12 del I Convenio y II Convenio y artículo 14 del III Convenio).

${ }^{49}$ Sin perjuicio de las referencias en los Convenios de Ginebra, se especifica en el artículo 38 Convención sobre los Derechos del Niño, adoptada por la Asamblea General de las Naciones Unidas el 20 de noviembre de 1989, en vigor desde el 2 de septiembre de 1990. Ratificado por España el 6 de diciembre de 1990, en vigor desde el 5 de enero de 1991 (BOE 313, 31 diciembre 1990).
} 
IUS ET SCIENTIA (ISSN: 2444-8478) 2019, Vol.5, nº 1, pp. 90-111

EXPERIMENTOS BIOLÓGICOS CON SERES HUMANOS EN EL MARCO DEL DERECHO DE LOS

CONFLICTOS ARMADOS, Ana Cristina GALLEGO HERNÁNDEZ Centro Universitario San Isidoro

(Universidad Pablo de Olavide). aagallegoo@gmail.com

Recibido: 14/01/2019. Aceptado: 16/05/2019 DOI: http://dx.doi.org/10.12795/IETSCIENTIA.2019.i01.04

Los conflictos armados, al igual que las situaciones de desastres naturales o en las catástrofes provocadas por el hombre, suelen conllevar consecuencias calamitosas de manera general y, en particular, un efecto devastador sobre la salud, generando en muchas ocasiones, incluso, brotes de enfermedades.

Entre los muchos casos y ejemplos, los cuales justifican la regulación actual, algunos de los experimentos biológicos, llevados a cabo en la Alemania del Tercer Reich consistían en realizar estudios sobre la congelación de seres humanos ${ }^{50}$. Ello "suponía la base científica para predecir mejor la reacción de los cuerpos ante el frío y poder emplear algunas variables para hacer más resistentes a los soldados ante esas condiciones" ${ }^{\text {"51 }}$ ya que un elevado porcentaje morían a causa de las circunstancias climatológicas. "Los conejillos de indias de estos experimentos inhumanos fueron jóvenes rusos y judíos”, es decir sujetos que pertenecían a grupos vulnerables y a los que no se les proporcionaba protección, que hoy el Derecho Humanitario plantean como imprescindible.

Sin ánimo de detallar casos tan espeluznantes, simplemente se pretende poner en relieve que, en situaciones de fuertes conflictos armados, algunos Estados han utilizado técnicas macabras para intentar sobrellevar los "contratiempos" en la batalla, por ejemplo, experimentando sobre las alturas para tener más éxitos con los paracaidistas o inyectando virus que comenzaban a afectar a batallones, con el fin de lograr la cura. Es por todo esto que para evitar el olvido de la ética profesional y personal, el derecho ha confeccionado todo el sistema jurídico de protección que ha sido descrito en los apartados anteriores y, al

\footnotetext{
${ }^{50}$ Además de los "crímenes de guerra médicos", como los denomina Mebring, del Tercer Reich -los cuales como es de común conocimiento han sido juzgados por el Tribunal Internacional de Núremberg- solo se ha juzgado otro caso por el Tribunal Internacional de Ruanda : Asunto Ntakirutimana en 2003 (por cerrar la consulta médica y negar el tratamiento a cienos de pacientes tutsi heridos por el conflicto). Esto demuestra que se ha desarrollado una muy escasa jurisprudencia y que los últimos conflictos que han ocasionado la creación de un Tribunal Internacional específico (Ruanda y Yugoslavia) ni contemplan los casos específicos de experimentos biológicos.

${ }^{51}$ REGADER, B., "Los experimentos con humanos durante el nazismo", Psicología y Mente. Disponible en: https://psicologiaymente.com/psicologia/experimentos-humanos-nazismo Consultado el 10 de octubre de 2018.
} 
IUS ET SCIENTIA (ISSN: 2444-8478) 2019, Vol.5, nº 1, pp. 90-111

EXPERIMENTOS BIOLÓGICOS CON SERES HUMANOS EN EL MARCO DEL DERECHO DE LOS

CONFLICTOS ARMADOS, Ana Cristina GALLEGO HERNÁNDEZ Centro Universitario San Isidoro

(Universidad Pablo de Olavide). aagallegoo@gmail.com

Recibido: 14/01/2019. Aceptado: 16/05/2019 DOI: http://dx.doi.org/10.12795/IETSCIENTIA.2019.i01.04

mismo tiempo, intenta ir delimitando las pautas a seguir en situaciones excepcionales o sobre las que no existe consenso.

Como se viene señalando, cuando en situaciones especiales (como son los conflictos armados y o brotes de enfermedades) resulte preciso y necesario realizar experimentos biológicos, sigue resultando fundamental que los principios éticos y el derecho que los recogen sigan cumpliéndose fielmente e incluso aumentando los controles exigidos. Ya que las investigaciones científicas en dichas situaciones inestables plantean retos importantes y urgentes ${ }^{52}$.

Evidentemente en dichos casos deberán respetarse cada una de las pautas exigidas en situaciones normales (consentimiento, información, necesidad, control de riesgos, resultados verificados, protección del participante, etc.). No obstante, las complicaciones se multiplican de manera que, si la investigación pretende dar solución a un hecho urgente o importante (un virus infeccioso) la revisión ética del procedimiento y del proyecto, deberá hacerse de manera acelerada. Ya que la vigilancia es un elemento trascendental para controlar que no se incumplen los códigos. Al mismo tiempo, estas causas, al mismo tiempo, pueden condicionar al reclutamiento de participantes, lo cual pone en entre dicho que se garantice la prestación libre de voluntad o de información. E incluso que los participantes hayan aceptado formar parte del estudio por presión. La urgencia empaña la transparencia.

Frente a los retos que una situación de conflicto armado puede generar, se pretende poner sobre la mesa, otros casos sobre los que ni derecho ni la ética han podido ofrecer

\footnotetext{
52 “Realizar una investigación en estas situaciones plantea retos importantes, como la necesidad de generar

conocimiento rápidamente, mantener la confianza pública y superar obstáculos prácticos a la ejecución de la investigación. Estos retos deben equilibrarse cuidadosamente con la necesidad de asegurar la validez científica de la investigación y mantener los principios éticos en su realización”. Consejo de Organizaciones Internacionales de las Ciencias Médicas y Organización Mundial de la Salud, "Pautas éticas internacionales para la investigación relacionada con la salud con seres humanos", Cuarta Edición. Ginebra: Consejo de Organizaciones Internacionales de las Ciencias Médicas (CIOMS); 2016, p. 83.
} 
IUS ET SCIENTIA (ISSN: 2444-8478) 2019, Vol.5, nº 1, pp. 90-111

EXPERIMENTOS BIOLÓGICOS CON SERES HUMANOS EN EL MARCO DEL DERECHO DE LOS

CONFLICTOS ARMADOS, Ana Cristina GALLEGO HERNÁNDEZ Centro Universitario San Isidoro

(Universidad Pablo de Olavide). aagallegoo@gmail.com

Recibido: 14/01/2019. Aceptado: 16/05/2019 DOI: http://dx.doi.org/10.12795/IETSCIENTIA.2019.i01.04

conductas general e internacionalmente aceptadas ${ }^{53}$. Se menciona un ejemplo; como se ha intentado explicar, principalmente, los Convenios de Ginebra determinan que los experimentos y/o amputaciones, sin justificación médica, son infracciones graves de dichos tratados y pueden ser calificados como crímenes de guerra, no obstante, al igual que bosqueja Sebring ${ }^{54}$, ¿están todas las amputaciones no justificadas, médicamente prohibidas? ¿Hay límites y excepciones? Los Estados, especialmente Occidentales, no persistimos en el intento de hacer ver a los pueblos que aún llevan a cabo prácticas, como la mutilación genital femenina, que incluso con el consentimiento (y deseo) de las partes afectadas, tienen como consecuencia la violación de derechos humanos. Diferente es que dichas amputaciones se realizaran sin el consentimiento, por la parte contraria de un conflicto y, evidentemente, sin que corresponda a necesidades de salud. En ese caso extremo sí que se ve claro el derecho a aplicar y las consecuencias jurídicas.

\section{Conclusiones}

El Derecho internacional Humanitario prohíbe que en situaciones de conflictos armados, ya sean internacionales o internos, se lleven a cabo experimentos biológicos o científicos sobre sujetos que ostentan una protección especial por los Convenios de Ginebra y sus Protocolos. No obstante esta prohibición deja abierta la delicada excepción a que la intervención médica se realice en protección de la salud y de conformidad con los principios éticos y jurídicos. Por ello, si como ocurría en la Alemania Nazi, la medicina fuese utilizada voluntariamente para ocasionar daños, miedo o para conseguir progresos científico sin tener en cuenta la dignidad humana, los autores de dichos actos serán juzgados, de conformidad con el Estatuto de Roma, por crímenes de guerra.

\footnotetext{
${ }^{53}$ Sin perjuicio de que la mayoría de Estados estipulen ciertas prácticas como violación de derechos fundamentales.

${ }^{54}$ En parecidos términos, MEBRING, S., op.cit., p. 248.
} 
IUS ET SCIENTIA (ISSN: 2444-8478) 2019, Vol.5, nº 1, pp. 90-111

EXPERIMENTOS BIOLÓGICOS CON SERES HUMANOS EN EL MARCO DEL DERECHO DE LOS

CONFLICTOS ARMADOS, Ana Cristina GALLEGO HERNÁNDEZ Centro Universitario San Isidoro (Universidad Pablo de Olavide). aagallegoo@gmail.com

Recibido: 14/01/2019. Aceptado: 16/05/2019 DOI: http://dx.doi.org/10.12795/IETSCIENTIA.2019.i01.04

De manera que, generalmente en situaciones de paz y ocasionalmente en conflicto armado, los experimentos médicos suponen prácticas imprescindibles para el continuo progreso, siempre que se justifique su necesidad y se realicen de conformidad con los principios de humanidad, imparcialidad y con las pautas establecidas en los códigos éticos.

En este sentido si la investigación médica está justificada por su necesidad, especialmente en situaciones de conflictos armados, se deberá prestar particular atención a que la práctica que se realice resulte coherente; a que los sujetos participantes (generalmente pertenecientes a grupos vulnerables: militares heridos y enfermos, prisioneros -de guerrao civiles, en particular si son enfermos, embarazadas, niños, discapacitados, etc.) hayan prestado el consentimiento libre de presiones; a que la información resulte ser clara, etc.

En definitiva, cabe la práctica de experimentos o investigaciones biológicas, pero siempre que el fin sea legítimo y cuando el objetivo sea coherente con la dignidad humana y el derecho internacional.

\section{Fuentes de consultadas}

- BADIA MARTÍ, A., “Asistencia humanitaria. Reflexiones desde la perspectiva jurídica internacional de los principios humanitarios y éticos", Revista Electrónica IUS ET SCIENTIA, no2, vol. 2, 2016.

- BADIA MARTÍ, A., "Comentarios a los "Principios éticos de la asistencia de salud en tiempo de conflictos armados y otras situaciones de emergencia", Comité Internacional Cruz Roja, 2015”, Revista de Bioética y Derecho \& Perspectivas Bioéticas, nº 36, 2016.

- Código de Núremberg. Experimentos médicos permitidos. Disponible en: http://www.bioeticanet.info/documentos/Nuremberg.pdf 
IUS ET SCIENTIA (ISSN: 2444-8478) 2019, Vol.5, nº 1, pp. 90-111

EXPERIMENTOS BIOLÓGICOS CON SERES HUMANOS EN EL MARCO DEL DERECHO DE LOS

CONFLICTOS ARMADOS, Ana Cristina GALLEGO HERNÁNDEZ Centro Universitario San Isidoro (Universidad Pablo de Olavide). aagallegoo@ gmail.com

Recibido: 14/01/2019. Aceptado: 16/05/2019 DOI: http://dx.doi.org/10.12795/IETSCIENTIA.2019.i01.04

- Consejo de Organizaciones Internacionales de las Ciencias Médicas y Organización Mundial de la Salud, "Pautas éticas internacionales para la investigación relacionada con la salud con seres humanos", Cuarta Edición. Ginebra: Consejo de Organizaciones Internacionales de las Ciencias Médicas (CIOMS); 2016.

- Convención sobre los Derechos del Niño, adoptada por la Asamblea General de las Naciones Unidas el 20 de noviembre de 1989, en vigor desde el 2 de septiembre de 1990. BOE 313, 31 diciembre 1990.

- Declaración de Helsinki de la AMM- Principios éticos para las investigaciones médicas en seres humanos. 64ª Asamblea General, Fortaleza, Brasil, octubre 2013.

- Estatuto de Roma de la Corte Penal Internacional, A/CONF.183/9, de 17 de julio de 1998. BOE 126, 27 de mayo de 2002.

- I Convenio de Ginebra para aliviar la suerte que corren los heridos y los enfermos de las fuerzas armadas en campaña, aprobado el 12 de agosto de 1949 por la Conferencia Diplomática para Elaborar Convenios Internacionales destinados a proteger a las víctimas de la guerra, celebrada en Ginebra del 12 de abril al 12 de agosto de 1949. Entrada en vigor: 21 de octubre de 1950.75 UNTS 31.

- IHL database, customary IHL. Disponible en: https://ihl-databases.icrc.org/customaryihl/eng/docs/v2_rul_rule92

- II Convenio de Ginebra para aliviar la suerte que corren los heridos, los enfermos y los náufragos de las fuerzas armadas en el mar, aprobado el 12 de agosto de 1949 por la Conferencia Diplomática para Elaborar Convenios Internacionales destinados a proteger a las víctimas de la guerra, celebrada en Ginebra del 12 de abril al 12 de agosto de 1949. Entrada en vigor: 21 de octubre de 1950. 75 UNTS 85.

- III Convenio de Ginebra relativo al trato debido a los prisioneros de guerra, aprobado el 
IUS ET SCIENTIA (ISSN: 2444-8478) 2019, Vol.5, nº 1, pp. 90-111 EXPERIMENTOS BIOLÓGICOS CON SERES HUMANOS EN EL MARCO DEL DERECHO DE LOS CONFLICTOS ARMADOS, Ana Cristina GALLEGO HERNÁNDEZ Centro Universitario San Isidoro (Universidad Pablo de Olavide). aagallegoo@gmail.com

Recibido: 14/01/2019. Aceptado: 16/05/2019 DOI: http://dx.doi.org/10.12795/IETSCIENTIA.2019.i01.04

12 de agosto de 1949 por la Conferencia Diplomática para Elaborar Convenios Internacionales destinados a proteger a las víctimas de la guerra, celebrada en Ginebra del 12 de abril al 12 de agosto de 1949. Entrada en vigor: 21 de octubre de 1950. 75 UNTS 135.

- IV Convenio de Ginebra relativo a la protección debida a las personas civiles en tiempo de guerra, aprobado el 12 de agosto de 1949 por la Conferencia Diplomática para Elaborar Convenios Internacionales destinados a proteger a las víctimas de la guerra, celebrada en Ginebra del 12 de abril al 12 de agosto de 1949. Entrada en vigor: 21 de octubre de 1950. 75 UNTS 287.

- MEBRING, S., “Medical war crimes”, Max Planck UNYB 15, 2011.

- Pacto Internacional de Derechos Civiles y Políticos (PIDCP), adoptado por la Asamblea General de las Naciones Unidas mediante la Resolución 2200 A (XXI), de 16 de diciembre de 1966. BOE $\mathrm{n}^{\circ}$ 103, de 30 de abril de 1977.

- Protocolo I adicional a los Convenios de Ginebra de 1949 relativo a la protección de las víctimas de los conflictos armados internacionales, 8 de junio de 1977. En vigor de forma general desde el 7 de diciembre de 1978. 1125 UNTS 3.

- Real Decreto 1090/2015, de 4 de diciembre, por el que se regulan los ensayos clínicos con medicamentos, los Comités de Ética de la Investigación con medicamentos y el Registro Español de Estudios Clínicos, BOE 307, 24 de diciembre de 2015.

- REGADER, B., "Los experimentos con humanos durante el nazismo", Psicología y Mente. Disponible en: https://psicologiaymente.com/psicologia/experimentos-humanosnazismo

- Resolución 808 (1993) del Consejo de Seguridad de 22 de febrero de 1993, aprobada en su $3175^{a}$ sesión, S/ RES/ 808 (1993). 
IUS ET SCIENTIA (ISSN: 2444-8478) 2019, Vol.5, nº 1, pp. 90-111

EXPERIMENTOS BIOLÓGICOS CON SERES HUMANOS EN EL MARCO DEL DERECHO DE LOS

CONFLICTOS ARMADOS, Ana Cristina GALLEGO HERNÁNDEZ Centro Universitario San Isidoro (Universidad Pablo de Olavide). aagallegoo@gmail.com

Recibido: 14/01/2019. Aceptado: 16/05/2019 DOI: http://dx.doi.org/10.12795/IETSCIENTIA.2019.i01.04

- Resolución 955 (1994) del Consejo de Seguridad de 8 de noviembre de 1994, aprobada en su $3453^{a}$ sesión, S/ RES/ 955 (1994).

- RODRÍGUEZ-VILLASANTE Y PRIETO, J. L., "Principios éticos de la asistencia sanitaria en tiempos de conflicto armado y situaciones de emergencia", Revista de Investigación y Educación en Ciencias de la Salud, nº 2, vol. 2, 2017.

- United Nations Audiovisual Library of International Law. Disponible en: http://legal.un.org/avl/pdf/ha/scsl/scsl_s.pdf 